\title{
Pseudo-Dynamic UWB WBAN Off-Body Radio Channel Measurements - Preliminary Results
}

\author{
Timo Kumpuniemi ${ }^{[0000-0003-4047-764 X]}$, Juha-Pekka Mäkelä, Matti Hämäläinen, \\ and Jari Iinatti \\ Centre for Wireless Communications, University of Oulu, Finland \\ timo.kumpuniemi@oulu.fi
}

\begin{abstract}
This paper presents measurement results on pseudo-dynamic ultra wideband off-body wireless body area network radio channels. The measurements are performed in an anechoic chamber in a 2-8 GHz frequency band by utilizing a vector network analyzer. A dynamic walking sequence was modeled by a test person who took five different body postures which were each measured statically. As a result, when observed together, the five postures can be used to model a dynamic walking sequence as in a cinema film. The antennas were attached on left and right wrist, and left ankle. The off-body antenna node was set on a pole. The work was repeated for two prototype antenna types: dipole and double loop. The mean attenuations of the first arriving paths were noted to lie between $-52 \ldots-68 \mathrm{~dB}$. No large differences were noted between the body postures. The link between left ankle and the pole had the largest attenuation. The averaged channel impulse response durations were noted to lie between eight to nine taps, where one tap corresponds to $0.167 \mathrm{~ns}$ in time. The dynamic range on the averaged link types shows values between $17 \ldots 28 \mathrm{~dB}$. No clear difference was noted in the performance between the antenna types.
\end{abstract}

Keywords: Ultra Wideband, Wireless Body Area Network, Channel Model.

\section{Introduction}

The fast progress in electric circuit technology has enabled increasing computing efficiency in constantly decreasing sizes with better energy efficiency simultaneously. At the same, also the battery technology has improved as well. As a result, small devices with low power consumption have become available in various applications that were not seen a few years ago. One hugely growing area is the internet of things (IoT) concept, which is one of the key areas in the fifth and sixth generation $(5 \mathrm{G}, 6 \mathrm{G})$ wireless systems. $5 \mathrm{G}$ is already in implementation phase and is expected to explode the IoT area and the number of sensors rapidly. $6 \mathrm{G}$ is currently under research in the academia and industry [1], and it is highly expected that after the deployment the boost on IoT will raise yet to another level.

With the IoT concept, wireless devices or sensors are, or are supposed to be, attached in or on various places in buildings, vehicles, industrial environment, machines and homes. They can monitor various parameters in their surroundings such as temperature, 
humidity, water leaks or motion. When several sensors are operating together, their data can be collected to a central node to be delivered further in the telecommunication network. Thus, the entity forms up a network, a concept often called as wireless sensor network (WSNs). Especially, when operating in a human, or even animal context, is very often called as wireless body area network (WBAN).

One of the most interesting applications areas for WSNs are particularly using them with animals or humans, as WBANs. The sensors can be attached on the surface of a person's body, a case called on-body communications. Some sensors may be located in the vicinity of a person but off the body surface, called often an off-body case. If a sensor or sensors are situated inside the body, it is called in-body communications. Communication between two or multiple persons each having in- or on-body sensors is often called body-to-body communications [2]. Also hybrid forms of this division may exist. The in-body sensor may communicate with a node at the body surface (inon- case) or with an off-body node (in-off case). The communication between on-body and off-body is sometimes called as an on-off body situation.

With respect to humans, several fields of usage can be found for WBANs. One quite popular is in the field of well-being and sports. The users can monitor their daily activity and the effectiveness of physical exercise both for professional athletes and fitness enthusiasts. The public sector users, e.g., firefighters, police officers and military troops may find WBANs very useful in increasing the safety in their professions.

The trend of increasing number of eldering people in many countries [3] brings another rapidly developing area for WBANs into the picture. It is the deployment of WBAN in the medical sector. The increasing portion of elderly people sets high demands to organize the healthcare to be available to all citizens at all times, not even to mention the exploding financial costs for the governments to provide these services. With WBANs, the workload and efficiency of medical staff can be eased up. The new technology enables remote monitoring of the patients leading their lives at their homes. The operations of hospitals and medical wards can be decentralized while the patients will be called into hospital facilities only when needed. The quality of life of the patients will be increased. Also the quality of medical treatment will be increased as the central vital parameters of the patients can be followed constantly through the sensors, instead of rarely conducted tests in a laboratory in centralized medical wards and hospitals.

Most solutions on WBANs nowadays are based on narrowband technologies operating most often at the Industrial, Scientific and Medical (ISM) frequency band at 2.45 $\mathrm{GHz}$ area. However, with many WBAN scenarios, the radio channels link distances are quite short. This is one reason, why the ultra wideband (UWB) technology is a very suitable solution for WBANs. UWB provides several other interesting features as well. It has a large bandwidth enabling a good performance in a harsh multipath environment. For the same reason, a precise positioning can be obtained, a feature finding several applications within the WBAN area. The tolerance against interference from other coexisting wireless systems is very high. On the other hand, the transmission power of UWB is low, creating minimum interference to other wireless systems, and a low exposure of electromagnetic radiation to humans, measured typically with the specific absorption rate. The transceiver structures are simple providing low unit costs and size, 
and their power consumption is low enabling long operation time when battery-operated $[4,5]$.

Real life application areas for WBANs using UWB in the medical field have been proposed, e.g., in [6], where a scenario for monitoring several vital parameters of a patient, e.g. an extensive care is explained. Another practical use case for WBANs is published in [7], which discusses the usage of UWB WBANs in the monitoring of patients suffering from Parkinson's disease.

UWB has been researched for WBANs for over ten years [8]. Quite many papers published are on the on-body communications cases, e.g., [9-11]. However, off-body cases with UWB are to found as well [12-16].

This paper shows preliminary results on pseudo-dynamic measurements in an offbody UWB WBAN scenario. The measured radio channels consist of the combined effect of propagation channels and antennas. The idea is to investigate effects due to the human body, especially on path losses, excluding other influences from the surroundings. For this purpose, the measurements are performed in an anechoic chamber. They are conducted at a 2-8 GHz bandwidth, using a vector network analyzer (VNA). Two prototype antenna types are used: dipole and double loop. The work is a part of a larger measurement campaign consisting of on-, off, and body-to-body cases. Additional results of the measurement campaign in static, pseudo-dynamic and truly dynamic cases are reported in, e.g., [6,10,14-18].

The structure of the paper is the following. In Section 2, the measurement setup is explained. Section 3 describes the measurement scenario with antenna locations and practical arrangements of the measurements. Section 4 explains the data processing methods together with the presentation of the results. Conclusions and future work plans are covered in Section 5.

\section{Measurement Setup}

\subsection{Test Person and the Measurement Environment}

A male test person at his late twenties was standing inside the anechoic chamber facing towards the off-body node. He was $183 \mathrm{~cm}$ tall with a body mass of $95 \mathrm{~kg}$. He was using a normal cotton T-shirt with jeans during the measurements. Shoes, belt, glasses, rings, jewelry or any other possibly metal containing artifacts were absent during the measurements.

The anechoic chamber had a floor size of $245 \mathrm{~cm}$ by $365 \mathrm{~cm}$. It was assembled by using a number of moving absorber blocks. It was located inside an electromagnetic compatibility room with a floor space of approx. 60 square meters, to avoid any excess radiation from other sources entering into the measurement area.

\section{$2.2 \quad$ Test Equipments}

A VNA with four ports of the type Rohde \& Schwarz ZVA-8 was applied in the measurements. It was located outside the anechoic chamber and connected inside the chamber with eight meter long measurement cables. The VNA was set to sweep the 2-8 GHz 
band in the frequency domain. 1601 frequency points were recorded, together with 100 sweeps for each measurements. Since a four-port VNA was applied with four antennas in use simultaneously, sixteen scattering parameters were obtained and all recorded. The sweep time was $288.18 \mathrm{~ms}$, with a $100 \mathrm{kHz}$ intermediate frequency bandwidth and $+10 \mathrm{dBm}$ transmission power.

The applied antennas were in-house built planar prototype antennas of two types: dipole and double loop. The maximum free space gains have been noted to be approx. $+6 \mathrm{dBi}$, and their operational bandwidth at 2-12 GHz. The reported performances and radiation patterns in free space of the antennas can be found in detail in [18-20].

\section{$3 \quad$ Measurement Scenario}

\subsection{Rationale for Pseudo-Dynamic Measurements}

Dynamic UWB WBAN radio channel measurements can be performed by using several methods in time or frequency domain. One very popular technique are frequency domain measurements with VNA as applied in this paper. One problem in dynamic measurements with VNA is the sweep time of the VNA, i.e., the time duration for one single frequency measurement across the frequency band set for the VNA. The sweep time depends on the quality of the VNA and the setting values for the measurements. In order to guarantee the validity of the measurements, the radio channel coherence time must be equal or longer than the sweep time of a measurement. There are several equations available in the literature for the channel coherence time $T_{0}$, out of which one of the strictest is defined by [21]

$$
T_{0}=9 /\left(16 \cdot \pi \cdot f_{d}\right),
$$

where $f_{d}$ is the Doppler frequency, defined as $f_{d}=v / \lambda$, where $v$ is the relative velocity between the antennas and is the $\lambda$ wavelength in the propagation media. Assuming propagation in air and the channel coherence time to be equal to sweep time of the VNA, the maximum relative velocity $v$ at exemplary frequencies of $2 \mathrm{GHz}$ and $8 \mathrm{GHz}$ (the measured bandwidth here) can be solved to be $v=9.3 \mathrm{~cm} / \mathrm{s}$ (at $2 \mathrm{GHz}$ ) and $v=2.3$ $\mathrm{cm} / \mathrm{s}$ (at $8 \mathrm{GHz}$ ). The latter sets the limit how fast a person could move in a dynamic measurement. In practice, the velocity should be lower to provide a safety margin between $T_{0}$ and the VNA sweep time. It is obvious from the example values, that the dynamic movement can be only very slow in order to guarantee the measurements to be valid, with the sweep time setting used in this work, making it difficult to carry out truly dynamic measurements in practice.

One solution to circumvent the problem is to use the pseudo-dynamic measurements approach, applied in the work in this article and previously in [17]. In it, several static measurements are conducted with different body postures. After combining the results in a sequence, a dynamic movement can be modelled as in a cinema film. 


\subsection{Antenna Placements}

The antennas were placed at the right wrist (RW), left wrist (LW) and left ankle (LA) on the body. The off body node (PO) was installed on a plastic pole at the height of 2 $\mathrm{m}$ and at the distance of $2 \mathrm{~m}$ from the test person. The locations are shown in Fig. 1. Combining the antenna position acronyms together, measured radio channels can be defined as the link between the left ankle and the pole (LA-PO), the link between the right wrist and the pole (RW-PO) and the link between the left wrist and the pole (LWPO). Three on-body antennas and the off-body node were measured each due to the four-port feature of the VNA. The on-body antennas were attached by using elastic bands and painter's masking tape. A $20 \mathrm{~mm}$ antenna-body distance was secured by the usage of ROHACELL HF31 [22] material between the antenna and the body. This distance was noted in [20] to provide good characteristics in antenna matchings and channel gain values simultaneously.

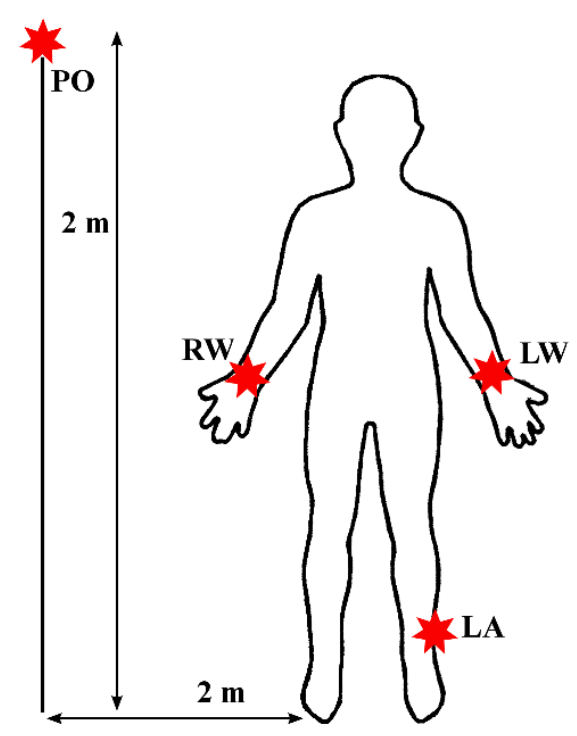

Fig. 1. The antenna locations used in the measurements.

\subsection{Pseudo-Dynamic Body Postures and Their Measurements}

The walking movement of two steps was emulated by the test person by starting from a standing position, facing directly to the pole. Due to practical restrictions in mobility set by the cables, the antennas and the anechoic chamber, the movements of limbs are limited in trajectory. Therefore they are imitating a walk of elderly people or people in a rehabilitation process after, e.g., an accident, a stroke, or a large surgical operation.

The walking sequence was modeled by five different body postures taken by the test person as shown in Fig. 2. At posture 1, the test person is standing at place. At posture 2 , a short step with his left foot is taken and his hands start to change position. At next step, posture 3 , the initiated move progresses by extending the trajectories of the left 
foot and the hands further. At postures 4 and 5, similar movement is repeated by starting the step with the left foot of the test person. In all postures, the distance to the pole was kept at two meters. In Fig. 2, also the antenna positions LW, RW, and LA are seen in practice.

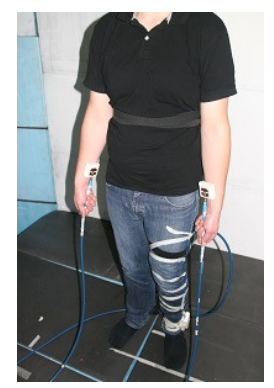

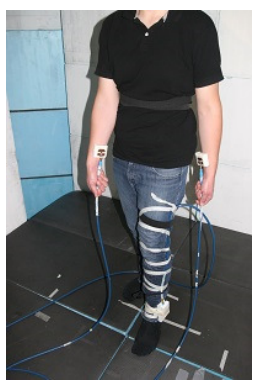

2

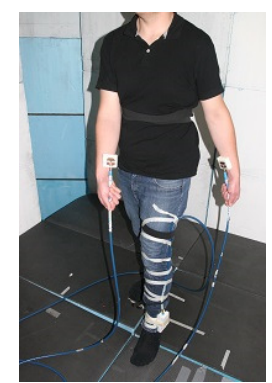

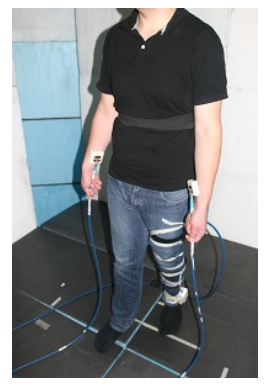

4

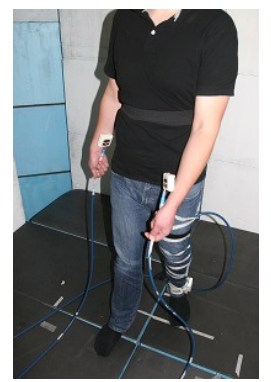

Fig. 2. Modelled pseudo-dynamic walking sequence.

In order to average out the possible variations in the body positions 1-5 in Fig. 2, they were measured five times. Both forward and reverse radio channels were measured. The same was repeated for antenna types (dipole and double loop). As a result, six off-body channels were recorded, resulting in total 15000 measured channel responses for both antenna types.

\section{Results}

\subsection{Data Processing}

The results are examined in the time domain by extracting the corresponding channel impulse responses (CIRs) from the raw measurement data. CIRs are obtained from the frequency domain data by applying the inverse fast Fourier transform algorithm (IFFT) in Matlab software. During the IFFT, no additional windowing was applied, in other words, it could be said, a rectangular windowing was used. 
The first arriving paths (FAPs) of the CIRs were observed as a start. Namely, it was noted that the multipath reflections occurring from the human body are decreasing rapidly in power compared to FAPs. In practical solutions, they should however be utilized in RAKE-type receivers. For this reason, also an investigation on CIR duration and the amplitudes of the other CIR taps beyond FAPs was performed. As easily understandable, the reflections originating beyond the human body are minor due to the anechoic chamber, but still visible revealing the unidealistic practical nature of the anechoic chamber.

To find out the FAP in every CIR, all multipath components were observed within a CIR. All components above a threshold value were solved, and the first one was selected to be the FAP. The threshold was set to lie $10 \mathrm{~dB}$ lower than the strongest component in a CIR, a value noted as a good choice in practice.

\subsection{Results of the First Arriving Paths}

Fig. 3 presents exemplary CIRs for one walking cycle with postures 1-5 for the link RW-PO using dipole antennas. It can be noted visually, that the attenuation of the CIRs with different postures varies by less than $5 \mathrm{~dB}$ giving the result that for the used scenario the human body does not shadow the signal with respect to the off-body antenna in the observed test person positions. The time delays of the FAPs vary due to the changing distance between the antennas depending on the position of the right wrist.

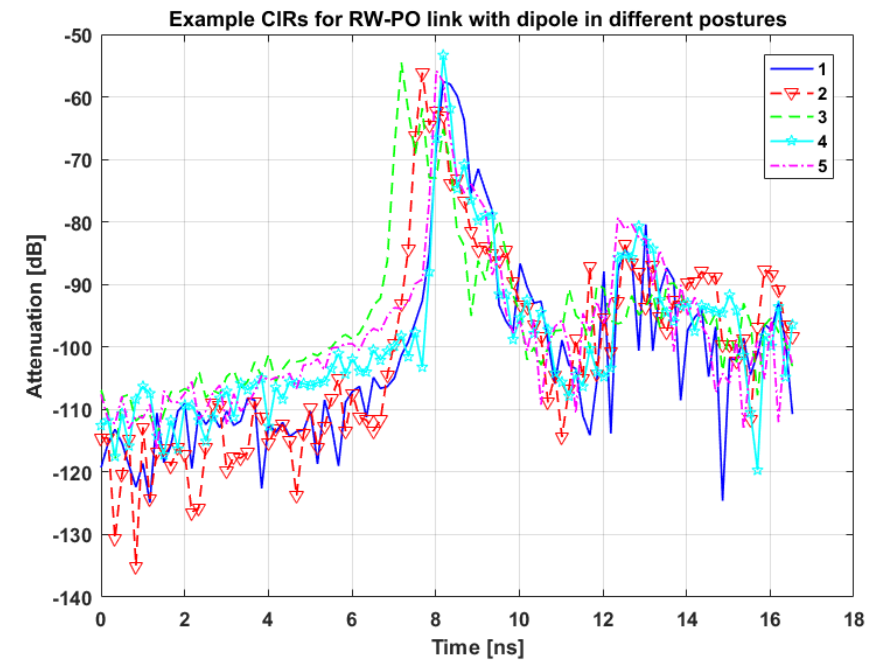

Fig. 3. Example CIRs for body postures 1-5 with RW-PO link using dipole.

To have an insight on the variation on the results between the five repetitions of the walking cycles, the FAP attenuations are next compared between one walking cycle and in the case when the results are averaged over five walking cycles for all off-body links and both antennas. The results are shown in Fig. 4 for one walking cycle and Fig. 
5 presents the case averaged over all five walking cycles. Several notifications can be made from Figs. 4 and 5.

Firstly, it can visually concluded, that there is no large difference when comparing the results of one walking cycle to the averaged result over five walking cycles. Therefore, the test person succeeded to repeat the body postures in a similar way in all cases.

Secondly, there is no large difference, generally speaking, between the links LW-PO and RW-PO. This is an understandable result as LW and RW are symmetric locations with respect to the off-body node. In most cases, the difference between LW-PO and RW-PO in attenuation remains within $1 \ldots 3$ decibels.

Thirdly, the LA-PO link shows the weakest performance, with attenuations up to 10 $\mathrm{dB}$ higher compared to the wrist links.

Fourthly, a comparison between the antenna types does not show a clear trend in attenuation and no firm conclusion can be made of the superiority between the antennas based on Figs. 4 and 5. This supports the results reported previously in, e.g., [6,10,14$18]$.
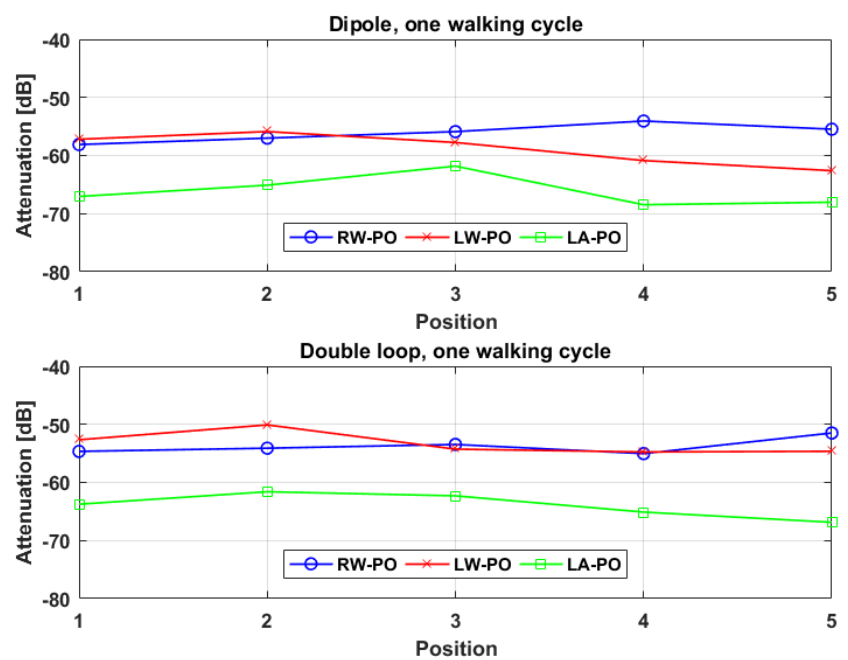

Fig. 4. Attenuation variation in one walking cycle. 

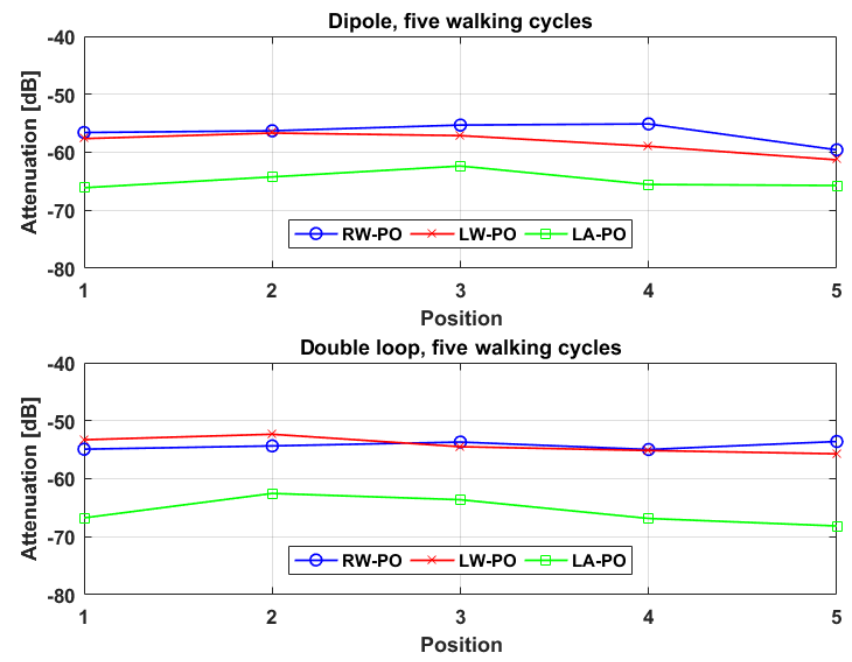

Fig. 5. Average attenuation variation in five walking cycles.

Table 1 collects the numerical data on FAPs when considering all five walking sequences measured. The average attenuations are shown, as well as the minimum and maximum values of them, for both antenna types. The following conclusions can be made.

The mean attenuation values between the different body postures within a single link type show quite small differences, within the range of approx. $1 \ldots 5 \mathrm{~dB}$. This describes that the applied measurement scenario with the test person facing directly to the pole, does not show a strong effect of body shadowing effect, when examining single radio links.

However, when comparing the different radio links, it is quite apparent, that the LA$\mathrm{PO}$ is the worst link in all positions and for both antenna types. LA-PO has typically approx. $10 \mathrm{~dB}$ higher attenuation compared to the LW-PO and RW-PO radio links. A probable reason to this is the relative position of the antennas. The left ankle antenna installation creates a higher body shadowing effect with respect to the off-body antenna. Furthermore, the relative antenna position of LA-PO is different than with the wrist links. As reported in [18], the antenna radiation patterns measured in free space show a high variation with respect to direction and frequency. Even though that the radiations patterns were not possible to be measured as on-body mounted, due to practical reasons, it is highly assumable that the same effect takes place in on-body cases as well.

When comparing the two antenna types with respect to the mean attenuation values, it can be concluded, that generally speaking, in 10 cases out of 15 , the double loop antenna shows better attenuation performance than the dipole. However, for the specific link of LA-PO, the dipole performs better in four cases out of five. However, the differences remain within few decibels only, giving a further verification that no clear difference between the antennas can be found out. 
The differences between the minimum and maximum attenuations of the separate links, antennas, and body postures lie between $3 \ldots 12 \mathrm{~dB}$. With the dipole, at RW-PO and position 5 , it has the maximum value $13.5 \mathrm{~dB}$. The variations can be explained by the understandable swaying characteristic of the test person while trying to stay in a fixed posture during the one measurement duration of 90 seconds. Furthermore, all measurements were repeated five times: even one sway of the test person in one measurement and position has a large numerical effect between the minimum and maximum values seen in Table 1 .

Table 1. Numerical attenuation results of the first arriving paths for five walking cycles.

\begin{tabular}{|c|c|c|c|c|c|c|c|}
\hline & & & \multicolumn{5}{|c|}{ Attenuation per position in $\mathrm{dB}$} \\
\hline & Link & Value & 1 & 2 & 3 & 4 & 5 \\
\hline \multirow{9}{*}{ 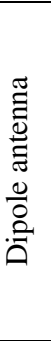 } & \multirow{3}{*}{ RW-PO } & Mean & -57 & -56 & -55 & -55 & -60 \\
\hline & & Min. & -53 & -55 & -54 & -53 & -54 \\
\hline & & Max. & -62 & -59 & -58 & -60 & -68 \\
\hline & \multirow{3}{*}{ LW-PO } & Mean & -58 & -57 & -57 & -59 & -62 \\
\hline & & Min. & -55 & -54 & -56 & -57 & -60 \\
\hline & & Max. & -60 & -60 & -60 & -63 & -66 \\
\hline & \multirow{3}{*}{ LA-PO } & Mean & -66 & -64 & -62 & -66 & -66 \\
\hline & & Min. & -65 & -63 & -61 & -64 & -65 \\
\hline & & Max. & -69 & -66 & -63 & -69 & -68 \\
\hline \multirow{9}{*}{ 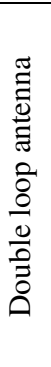 } & \multirow{3}{*}{ RW-PO } & Mean & -55 & -54 & -54 & -55 & -54 \\
\hline & & Min. & -54 & -53 & -52 & -53 & -51 \\
\hline & & Max. & -58 & -57 & -58 & -59 & -59 \\
\hline & \multirow{3}{*}{ LW-PO } & Mean & -53 & -52 & -55 & -55 & -56 \\
\hline & & Min. & -50 & -50 & -51 & -53 & -54 \\
\hline & & Max. & -59 & -58 & -59 & -61 & -60 \\
\hline & \multirow{3}{*}{ LA-PO } & Mean & -67 & -63 & -64 & -67 & -68 \\
\hline & & Min. & -62 & -61 & -61 & -64 & -66 \\
\hline & & Max. & -72 & -64 & -66 & -70 & -70 \\
\hline
\end{tabular}

\subsection{Results of the CIRs}

This chapter explains the length of the CIRs reasonable to observe as well as the average attenuations for each CIR tap. In this paper, the concept of CIR tap is defined to be one single sample in the CIR, i.e., the time separation between two consecutive taps is the inverse of the measurement bandwidth, $\Delta t=(1 / 6 \mathrm{GHz}) \approx 0.167 \mathrm{~ns}$. The CIR channel taps therefore are not sparse in time.

As noted with the exemplary CIRs presented in Fig. 3, the CIRs have a tendency to decay quite rapidly in anechoic chamber measurements. The threshold to define the limit for CIR taps of interest is defined as follows. Considering the IEEE802.15.6 standard [23], it states that an impulse radio UWB receiver should have a minimum sensitivity of $-91 \mathrm{dBm}$, assuming a receiver noise figure of $10 \mathrm{~dB}$ and an implementation loss of $5 \mathrm{~dB}$. 
Federal Communications Commission (FCC) from its side sets a maximum transmit power spectrum density limit for UWB in a 3.1-10.6 GHz band to be $-41.3 \mathrm{dBm} / \mathrm{MHz}$ [4]. For one UWB channel with a bandwidth of $499.2 \mathrm{MHz}$ set in [23], it corresponds to a power of $-14.3 \mathrm{dBm}$. Let us assume an optimistic extra margin of $5 \mathrm{~dB}$ for the development in receiver noise figures and implementation. Then, it yields that the CIR attenuation values below $-82 \mathrm{~dB}$ give only a small contribution to the total signal power, assuming RAKE-receivers, in the scenario under investigation here.

Fig. 6 presents an example of the definition of CIR length for links RW-PO, LWPO and LA-PO for dipole antenna, at body posture 1 . They are solved as as the average over all five measurement repetitions, all frequency sweeps and both forward and reverse channels. The black vertical line is the $-82 \mathrm{~dB}$ threshold level. Tap number one is the FAP. The CIR length is defined to be the number of taps when the CIR first time falls below the threshold. Therefore, in Fig. 6, the CIR lengths are 5 (LA-PO), and 6 (RW-PO, LW-PO) taps.

Similar investigation are next repeated for each link, body position and for both antenna types. The results are gathered in Table 2. As noted, the CIR tap lengths vary between five to ten CIR taps. Comparison between link types reveals that on the average LA-PO has one tap shorter CIR duration than the wrist links for both antennas. This is naturally due to the higher attenuation of LA-PO noted in Table 1 as well. Comparison between the antenna types shows, that on the average the CIR tap numbers are 7.0 and 7.2 for the dipole and double antennas. With this respect, no clear performance difference between the antennas can be noted.

As the next step, the average attenuations per each tap are presented for each link and antenna separately. As noted in Fig. 3, the decay slope in the exemplary case is basically body position independent. Therefore, we average over all body positions to obtain link and antenna specific data to obtain a reasonable number of numerical values to be presented. 


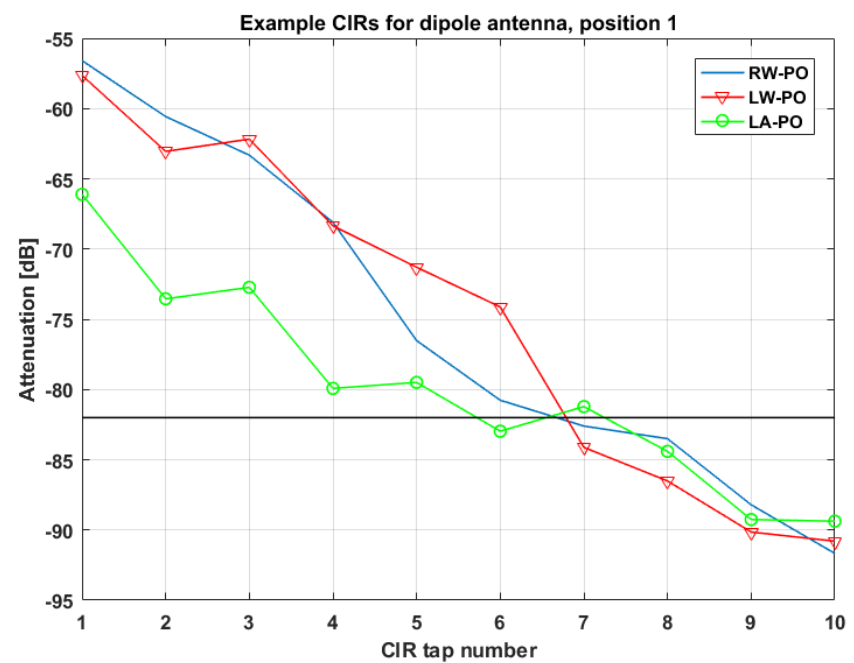

Fig. 6. Example CIR lengths for different links with dipole, body position 1.

Table 2. CIR lengths per link and per body posture.

\begin{tabular}{|c|c|c|c|c|c|c|}
\hline & & \multicolumn{5}{|c|}{ CIR length in taps per body posture } \\
\hline & Link & 1 & 2 & 3 & 4 & 5 \\
\hline \multirow{3}{*}{$\begin{array}{l}\frac{0}{0} \\
\stackrel{\circ}{0}\end{array}$} & RW-PO & 6 & 8 & 9 & 6 & 7 \\
\hline & LW-PO & 6 & 6 & 7 & 8 & 10 \\
\hline & LA-PO & 5 & 7 & 8 & 6 & 6 \\
\hline \multirow{3}{*}{$\frac{\frac{0}{0}}{\frac{0}{0}}$} & RW-PO & 6 & 9 & 9 & 7 & 7 \\
\hline & LW-PO & 7 & 7 & 7 & 8 & 9 \\
\hline & LA-PO & 8 & 5 & 5 & 7 & 8 \\
\hline
\end{tabular}

Table 3 collects the results with respect to the dipole antennas. As can be noted, the CIR tap lengths in the averaged case extend to seven or eight taps. Due to the higher FAP attenuation in LA-PO links, it has the shortest CIR duration.

Table 4 shows the corresponding results with the double loop antennas. The difference with the dipole antenna is minor, showing only a one tap longer CIR response with links RW-PO and LA-PO.

Finally, one interesting parameter may sometimes the available dynamic range of a radio link. The results are obtained by solving the difference between the first CIR tap attenuations from Table 3 and Table 4 and the applied detection threshold level of -82 $\mathrm{dB}$. The dynamic range lies between $24 \ldots 28 \mathrm{~dB}$ for the wrist links and at $17 \mathrm{~dB}$ for LA-PO. In two cases out of three the double loop antennas reaches a better performance 
than the dipole. In practice, the performance with LA-PO is similar, and also the difference in RW-PO and LA-PO is $2 \ldots 4 \mathrm{~dB}$.

Table 3. Average attenuation per CIR taps per link for the dipole antenna.

\begin{tabular}{llll}
\hline & RW-PO & LW-PO & LA-PO \\
\hline CIR tap & Attenuation [dB] & Attenuation [dB] & Attenuation [dB] \\
1 & -56 & -58 & -65 \\
2 & -62 & -65 & -73 \\
3 & -66 & -65 & -73 \\
4 & -67 & -67 & -77 \\
5 & -73 & -69 & -80 \\
6 & -75 & -72 & -80 \\
7 & -79 & -78 & -81 \\
8 & -81 & -81 & - \\
\hline
\end{tabular}

Table 4. Average attenuation per CIR taps per link for the double loop antenna.

\begin{tabular}{llll}
\hline & RW-PO & LW-PO & LA-PO \\
\hline CIR tap & Attenuation $[\mathrm{dB}]$ & Attenuation $[\mathrm{dB}]$ & Attenuation $[\mathrm{dB}]$ \\
1 & -54 & -54 & -65 \\
2 & -60 & -60 & -70 \\
3 & -62 & -63 & -72 \\
4 & -65 & -65 & -73 \\
5 & -67 & -71 & -77 \\
6 & -72 & -73 & -77 \\
7 & -75 & -79 & -80 \\
8 & -80 & -81 & -82 \\
9 & -81 & - & - \\
\hline
\end{tabular}

Table 5. Average available dynamic range per link and per antenna in $\mathrm{dB}$.

\begin{tabular}{llll}
\hline & RW-PO & LW-PO & LA-PO \\
\hline Dipole & 26 & 24 & 17 \\
Double loop & 28 & 28 & 17 \\
\hline
\end{tabular}




\section{$5 \quad$ Conclusion and Future Work}

Pseudo-dynamic UWB WBAN off-body radio are discussed in this paper. The results are preliminary in nature, as only one test person position with respect to the off-body pole is considered. The measurements are performed in an anechoic chamber using a VNA within the frequency range of 2-8 GHz. Pseudo-dynamic approach was used to model a slow dynamic walking sequence. The research is repeated with prototype dipole and double loop antennas.

Considering firstly the first arriving paths of the CIRs, it was noted that their averaged attenuations lied between $-52 \ldots-68 \mathrm{~dB}$ depending on the body posture, radio channel link, and antenna type. No large differences were noted between the body postures. Considering the channel impulse response durations, they were noted to lie between five to ten taps when examining each link and body posture separately. After averaging the CIR duration data all body postures, the CIR lengths were noted to be eight to nine taps. No clear difference was noted in the performance between the antenna types. The obtained dynamic range within the averaged links vary between $17 . . .28 \mathrm{~dB}$. LA-PO has clearly lower performance compared to RW-PO and LW-PO. Comparison between the antennas shows quite similar performance between the antennas in two links (RW-PO and LA-PO).

As per the future work, a larger variety of scenarios would be interesting to measure. This includes firstly different relative positions of the test person to the off-body pole. Secondly, radio channels in echoic environments should be measured as well.

\section{Acknowledgement}

This research has been financially supported in part by Academy of Finland 6Genesis Flagship (grant 318927), and in part by the project WBAN communications in the congested environment (MeCCE).

\section{References}

1. 6Genesis Flagship Homepage, https://www.oulu.fi/6gflagship/, last accessed 2019/06/28.

2. Hall, P.S., Hao, Y.: Antennas and propagation for body-centric wireless communications. 2nd edn. Artech House, Norwood, MA, USA (2012).

3. United Nations, Department of Economic and Social Affairs, World Population Ageing 2017 (accessed in August 2019), [Online]. Available: http://www.un.org/en/development/desa/population/ publications/pdf/ageing/WPA2017_Highlights.pdf.

4. Oppermann, I., Hämäläinen, M., Iinatti, J. (eds.): UWB theory and applications. John Wiley $\&$ Sons, West Sussex, England (2004).

5. Ghawami, M., Michael, L.B., Kohno, R.: Ultra wideband signals and systems in communication engineering, 2nd edn. John Wiley \& Sons, West Sussex, England (2007).

6. Kumpuniemi, T., Hämäläinen, M., Yekeh Yazdandoost, K., Iinatti, J.: Categorized UWB onbody radio channel modeling for WBANs. Progress in Electromagnetic Research B, 67, 1-16 (2016). 
7. Keränen, N., Särestöniemi, M., Partala, J., Hämäläinen, M., Reponen, J., Seppänen, T., Iinatti, J., Jämsä, T.: IEEE 802.15.6-based multi-accelerometer WBAN system for monitoring Parkinson's disease. In: Proceedings of the $35^{\text {th }}$ Annual International Conference of the IEEE engineering in medicine and biology society (EMBC), 1656-1659, IEEE, Osaka, Japan (2013).

8. Fort, A., Desset, C., De Doncker, P., Wambacq, P., Van Biesen, L:, An ultra-wideband body area propagation channel model-from statistics to implementation. IEEE Transactions on Microwave Theory and Techniques, 54(4), 1820-1826 (2006).

9. Di Bari, R., Abbasi, Q. H., Alomainy, A., Hao, Y.: An advanced UWB channel model for body-centric wireless networks. Progress In Electromagnetics Research, 136, 79-99 (2013).

10. Kumpuniemi, T., Hämäläinen, M., Yekeh Yazdandoost, K, Iinatti, J.: Human body shadowing effect on dynamic UWB on-body radio channels. IEEE Antennas and Wireless Propagation Letters, 16, 1871-1874 (2017).

11. Ali, A. J., Scanlon, W. G., Cotton, S. L.: Pedestrian effects in indoor UWB off-body communication channels. In: Proceedings of. 2010 Loughborough Antennas \& Propagation Conference, 57-60, IEEE, Loughborough, UK (2010).

12. Catherwood, P. A., Scanlon, W. G.: Body-centric antenna positioning effects for off-body UWB communications in a contemporary learning environment. In: Proceedings of the 8th European Conference on Antennas and Propagation (EUCAP), 1571-1574, IEEE, The Hague, Netherlands (2014).

13. Garcia-Serna, R.-G., Garcia-Pardo, C., Molina-Garcia-Pardo, J.: Effect of the receiver attachment position on ultrawideband off-body channels. IEEE Antennas and Wireless Propagation Letters, 14, 1101-1104 (2015).

14. Kumpuniemi, T., Mäkelä, J-P., Hämäläinen, M., Yekeh Yazdandoost, K., Iinatti, J.: Dynamic UWB off-body radio channels - human body shadowing effect. In: Proceedings of the 28th Annual IEEE International Symposium on Personal, Indoor and Mobile Radio Communications (IEEE PIMRC 2017), 1-7, IEEE, Montreal, Canada (2017).

15. Kumpuniemi, T., Mäkelä, J-P., Hämäläinen, M., Yekeh Yazdandoost, K., Iinatti, J.: Human body effect on static UWB WBAN off-body radio channels. In: Proceedings of the 13th EAI International Conference on Body Area Networks (BODYNETS 2018), 1-10, EAI, Oulu, Finland (2018).

16. Kumpuniemi, T., Mäkelä, J-P., Hämäläinen, M., Yekeh Yazdandoost, K., Iinatti J.: Measurement and analysis on dynamic off-body radio channels at UWB frequencies. In: Proceedings of the 13th International Symposium on Medical Information and Technology (ISMICT 2019), 1-5, IEEE, Oslo, Norway (2019).

17. Kumpuniemi, T., Hämäläinen, M., Tuovinen, T., Yekeh Yazdandoost, K., Iinatti J.: Radio channel modelling for pseudo-dynamic WBAN on-body links. In: Proceedings of the 8th International Symposium on Medical Information and Technology (ISMICT 2014), 1-5, IEEE, Florence, Italy (2014).

18. Kumpuniemi, T., Hämäläinen, M., Yekeh Yazdandoost, K., Iinatti, J.: Measurements for body-to-body UWB WBAN radio channels. In: Proceedings of the 9th European Conference on Antennas and Propagation (EUCAP), 1-5, IEEE, Lisbon, Portugal (2015).

19. Tuovinen, T., Kumpuniemi, T., Yekeh Yazdandoost, K., Hämäläinen, M., Iinatti, J.: Effect of the antenna-human body distance on the antenna matching in UWB WBAN applications. In: Proceedings of the $7^{\text {th }}$ International Symposium on Medical Information and Communication Technology (ISMICT), 193-197, IEEE, Tokyo, Japan (2013).

20. Tuovinen, T., Kumpuniemi, T., Hämäläinen, M., Yekeh Yazdandoost, K., Iinatti, J.: Effect of the antenna-body distance on the on-ext and on-on channel link path gain in UWB WBAN applications. In: Proceedings of the 35th Annual International Conference of IEEE Engineering in Medicine and Biology Society (EMBC), 1242-1245, IEEE, Osaka, Japan (2013). 
21. Saunders, S.R., Aragón-Zavala, A: Antennas and propagation for wireless communication systems. 2nd edn. John Wiley \& Sons, Chichester, West Sussex, England, (2007).

22. Rohacell Homepage, https://www.rohacell.com/product/rohacell/en/products-services/rohacell-hf/, last accessed 2019/06/28.

23. IEEE Standard for Local and Metropolitan Area Networks, IEEE 802.15.6-2012-Part 15.6: Wireless Body Area networks, 2012. 